\title{
Temperature Profiling Femtosecond Laser Induced Modifications Through Transmission Electron Micrscopy
}

\author{
Y.N. Picard,* S.M. Yalisove, ${ }^{*}$ \\ * Department of Materials \& Engineering, University of Michigan, 2300 Hayward St., \\ Ann Arbor, MI 48109
}

Femtosecond pulse-length lasers continue to attract interests in nano-scale material removal and modification. Such interests in ultra-short pulsed lasers stem from the limited energy dissipation following laser-matter interaction. Improving spatial resolution of direct laser machining requires better understanding of the nature and extent of any modifications induced in the material by the laser.

We demonstrate a method for assessing material modifications induced by single femtosecond laser pulses at fluences sufficient for ablation using TEM and a multilayer material system. The Co-Si system consists of multiple stable phases with various formation temperatures and could prove useful for determining maximum temperatures reached adjacent to laser irradiated regions. In this study, a $\mathrm{Co} / \mathrm{Si}$ thin film system was deposited and laser irradiated by femtosecond laser pulses. Extent of modification induced by single pulses was directly observed using TEM imaging and selected area electron diffraction (SAED).

Co and $\mathrm{Si}$ were deposited on $\mathrm{Si}$ (100) substrates at room temperature using a molecular beam epitaxy (MBE) system at a base pressure of $5 \times 10^{-11}$ Torr. A 20-300 $\AA$ thick film of cobalt was deposited, followed immediately by deposition of $200 \AA$ silicon. A series of single pulses from a Ti:sapphire femtosecond laser system was exposed to the samples towards the film side to produce a grid of laser holes at a spacing of 20 microns (Figure 1A). Transmission electron microscopy was used to image and analyze the structure of the area adjacent to the laser holes. We used a Phillips CM-12 for lower resolution analysis and a JEOL 3011 for higher resolution and selected area electron diffraction (SAED) analysis.

In this work, we observed two zones of modification induced by a single pulse at a laser fluence of $0.9 \mathrm{~J} / \mathrm{cm}^{2}$. The first zone extended from the very edge of the ablate hole to within 1 micron away. In this zone is evidence of elongated crystallized silicon (Figure 2 ), indicative of temperatures reaching that of the melting temperature of silicon. A second zone extends 1-3 microns from the ablated hole edge, where evidence of $\mathrm{CoSi}_{2}$ formation is observed (Figure 1B). Further away is little evidence of any futher modification (Figure 1C). Hence, we can conclude that significant heating occurred outside of the laser ablated region upon irradiation but only to within 3 microns. In addition, the rate of dissipation was slow enough to allow for silicon crystallization, potentially due to the constricted heat conduction path of the thin foil. 


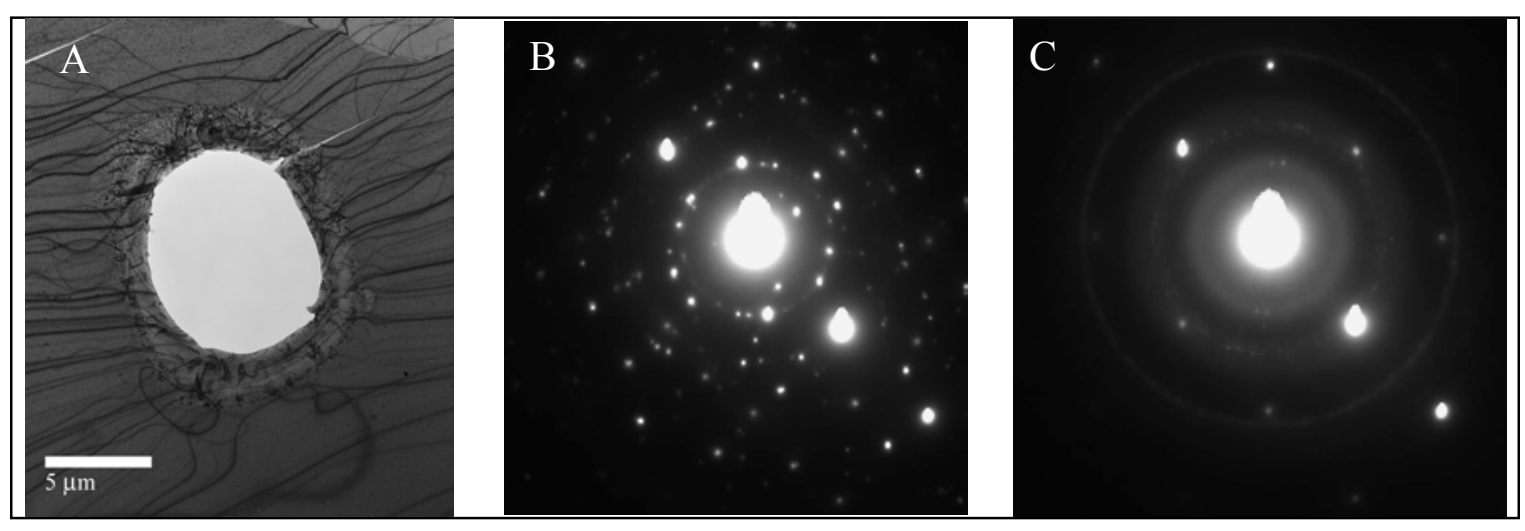

Fig. 1. A. Bright field transmission electron micrograph of a femtosecond laser ablated hole in $\mathrm{Co} / \mathrm{Si}$ multilayer. Selected area electron diffraction patterns taken B. 1.6 microns and C. 3.2 microns away from the ablated hole edge.

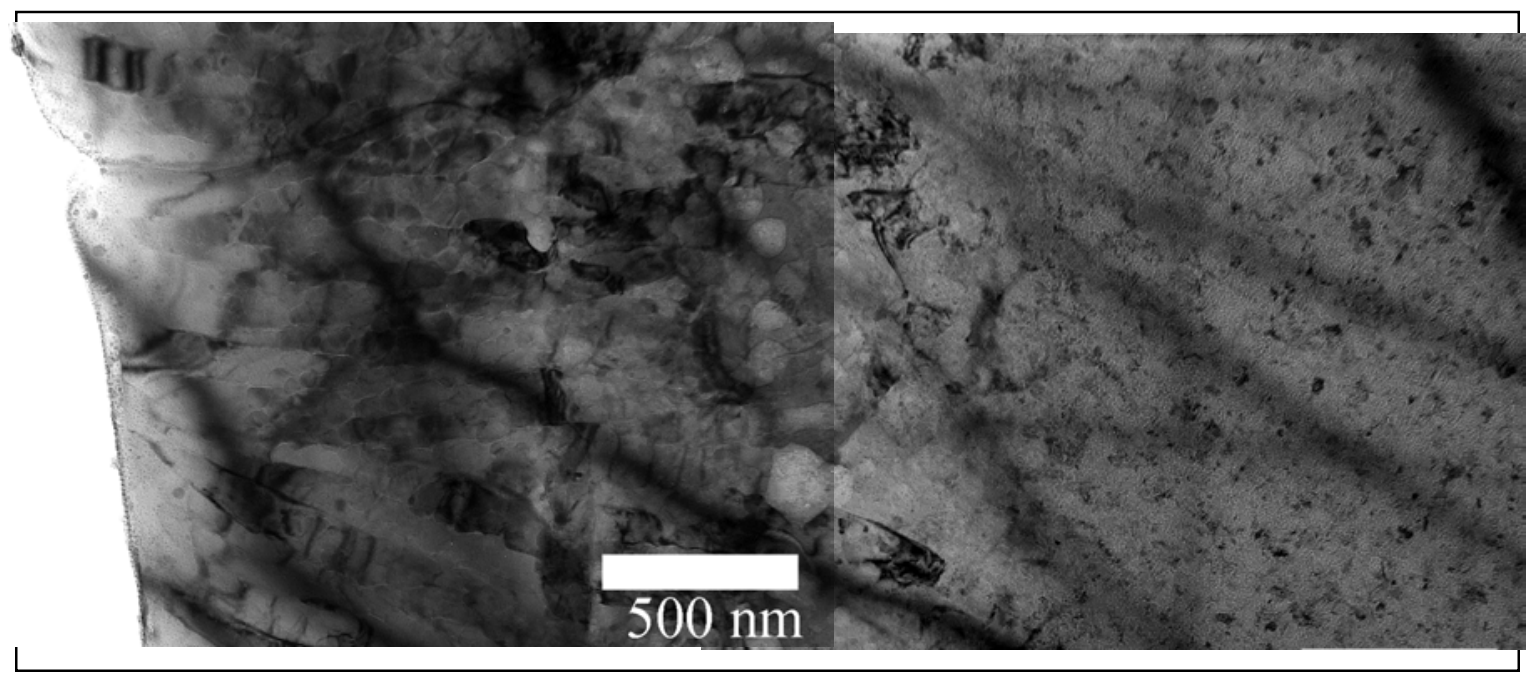

Fig. 2. Bright field transmission electron micrographs of the laser ablated hole edge. 